\title{
Nuclear winter can cross Equator
}

\section{A new calculation shows that smoke from nuclear conflagrations will reach the Southern Hemisphere from a northern nuclear war, especially in the northern summer. But many other problems persist.}

How certain is the picture built up in the past few months of the nuclear winter that would follow a major exchange of nuclear weapons? Later this month, the group appointed by the Scientific Committee on Problems of the Environment (SCOPE) of the International Council of Scientific Unions to brood about the nuclear winter and the other consequences of nuclear war will be making public its first report; by all accounts, it will be more a judicious assessment of what remains to be done to define the uncertainties in the problem than a prescription of what the nuclear winter would be like, which is entirely appropriate.

Meanwhile, the article by Stanley $\mathbf{L}$. Thompson on p. 35 of this issue will help to fill one important if small gap in previous arguments: smoke caused by a nuclear war between combatants in the Northern Hemisphere will rise in altitude faster, and spread more quickly to the Southern Hemisphere, than smoke from a nuclear war in the north during the northern winter. The conclusion is what would be expected from crude handwaving; the explanation is largely that insolation energizes the atmosphere predominantly in the summer hemisphere. But since the interhemispheric transfer of smoke was of necessity neglected in the original calculations, and because its distribution affects the expected changes of temperature supposed to lead to nuclear winter, it is valuable that the point has been established by a strictly global threedimensional model of the atmosphere.

Readers will also note that Thompson, like others working in this field, lists several points on which it appears that the first accounts of nuclear winter must in due course be refined. One of the most obvious of these is also one of the most inaccessible - the estimates that are used for the quantities of smoke lofted into the atmosphere after the conflagrations likely to be started by a general exchange of nuclear weapons. It is generally agreed that urban and rural fires will be respectively more and less effective generators of smoke, but there is much more scope for argument about the quantities likely to be produced by general urban conflagrations. One difficulty is that the issue cannot, by definition, be resolved by experiment; even conventionally started fires would be pale imitations of those likely to follow nuclear attacks on cities, while the few historical opportunities for observa- tions (as at Dresden and Tokyo towards the end of the Second World War) have been lost. Moreover, to the extent that the severity of a nuclear winter would be directly determined by the amount of smoke released into the atmosphere in a nuclear exchange, the course of a postattack winter is bound to be a sensitive function of the exact pattern of the attack.

Thompson also draws attention to the need for a better understanding of the way in which some part of the smoke generated by large fires would be promptly removed by the clouds they necessarily generate. This mechanism was part of the reason given by Dr Edward Teller last year for scaling down the original predictions of nuclear winter by R.P. Turco et al. (Science 222, 1283; 1983). It would be surprising if large fires prove to be in some sense self-limiting in their consequences for direct insolation, but there is equally no obvious reason why effect and cause should be arithmetically proportional to each other. Again this is a point which is almost impossible to check directly.

Still another difficulty, which Thompson does not on this occasion list, is that of knowing what are the consequences of the inevitable patchiness in the cloud of smoke from several nuclear conflagrations, at least in the early stages in the evolution of a nuclear winter. Originally, Turco et al. supposed that several days would pass before their cloud of smoke was uniformly dispersed over some band of latitude. Put crudely, the difficulty is that of estimating the climatic consequences of two neighbouring vertical sections of the atmosphere, one normal, the other in which the vertical flux of solar radiation is much reduced by the effects of smoke in the upper troposphere.

Calculations cannot be straightforward, given that the system is far from equilibrium. But it is entirely possible that two such adjacent slices of the atmosphere would function as a means of carrying water vapour high into the atmosphere, so as to form clouds that would then remove smoke. But even if that were possible, precisely what happened would no doubt depend sensitively on the nature of the underlying terrain, sea surface or dry land.

Merely to list uncertainties such as these, among which are the difficulties of including real clouds rather than average cloudiness in climatic models, is not to demonstrate that nuclear winter is an illusion. At this stage, there is probably no reason to dissent from the statement in Thompson's article that the uncertainties in calculating nuclear winter allow effects that range from "mild local cooling to subfreezing temperatures on a global scale".

The immediate consequences of the likelihood that a major nuclear war, involving the use of a substantial fraction of the world's nuclear dedicated nuclear explosives, are similarly problematical. It has sometimes been argued that the possibility of nuclear winter of such a severity that most living things in at least one hemisphere would be killed argues for a reduction of nuclear stockpiles below whichever threshold may make such a catastrophe possible. But that is not a unique argument for arms control agreements that will effect a reduction of nuclear arsenals on such a scale; people's fond wish to sleep easily at night is a sufficient case for that.

Others say that the severity of the standard nuclear winter as first described by Turco et al. makes nonsense of existing arrangements for dealing with some of the consequences of nuclear war, plans for building fallout shelters, for example. In reality, the validity of this argument is more restricted. Even if the predictions of the kind of nuclear winter that would follow an all-out exchange of nuclear weapons are correct, the assumption that all nuclear wars must be all-out wars is surely mistaken. On at least some credible opinions about the course of events that might lead to nuclear war, the first steps would entail the use of small numbers of nuclear weapons, perhaps tactically in places such as central Europe. The function, misguided though it may be, of such hostile acts would be to extend a threat of seriousness to an opponent in time to force second thoughts, for which purpose just one or two weapons might suffice. Fallout shelters may have some value in those circumstances.

The implications of all this are simply that there is no obvious way in which the concept of nuclear winter affects strategy and international relations in ways that are unique. Non-nuclear weapons states such as India may rightly claim that the prospectus threatens them when they might expect to escape direct consequences of a major nuclear war between major nuclear powers, but that is only a part (and a recent part) of their traditionally stolid attitude on the need for strategic nuclear arms control.

John Maddox 